\title{
Evidence of increasing recorded diagnosis of autism spectrum disorders in Wales, UK: An e-cohort study
}

Autism

$1-10$

(C) The Author(s) 2021

(c) (i)

Article reuse guidelines:

sagepub.com/journals-permissions

DOI: 10.1 I77/|36236|32|I059674

journals.sagepub.com/home/aut

(S)AGE

\author{
Jack FG Underwood' (D), Marcos DelPozo-Banos², \\ Aura Frizzati ${ }^{3}$, Ann John ${ }^{2}$ and Jeremy Hall'
}

\begin{abstract}
Estimates place the prevalence of autism spectrum disorders (autism) at around I\% in the population. New services for adult diagnosis have been set up in Wales, UK, at a time of rising awareness of the spectrum of autism experiences; however, no studies have examined adult autism prevalence in Wales. In this study, we used an anonymised e-cohort comprised of healthcare record data to produce all-age estimates of prevalence and incidence of recorded autism for the years 200 I-20I6. We found the overall prevalence rate of autism in healthcare records was $0.5 \mathrm{I} \%$. The number of newrecorded cases of autism increased from 0.188 per 1000 person-years in 2001 to 0.644 per 1000 person-years in 2016. The estimate of $0.51 \%$ prevalence in the population is lower than suggested by population survey and cohort studies study methodologies, but comparable to other administrative record study estimates. Rates of new incident diagnoses of autism saw a $>150 \%$ increase in the years 2008-2016, with a trend towards more diagnoses in those aged over 35years and an eightfold increase in diagnoses in women from 2000 to 2016 . This study suggests that while the number of people being diagnosed with autism is increasing, many are still unrecognised by healthcare services.
\end{abstract}

\section{Lay abstract}

Autism spectrum disorders (autism) are thought to be relatively common, with analyses estimating $1 \%$ in the population could meet diagnostic criteria. New services for adult diagnosis have been set up in Wales, UK; however, no studies have examined for the proportion of adults with autism in Wales. In this study, we take anonymised healthcare record data from more than 3.6 million people to produce a national estimate of recorded autism diagnoses. We found the overall prevalence rate of autism in healthcare records was $0.51 \%$. The number of new-recorded cases of autism increased from 0.188 per 1000 person-years in 2001 to 0.644 per 1000 person-years in 2016 . The estimate of $0.51 \%$ prevalence in the population is lower than suggested by population survey and cohort studies, but comparable to other administrative records. From 2001 to 2016 , the number of autism services for adults has increased, and autism is more widely known in society, while concurrently in healthcare records, there was a $>150 \%$ increase autism diagnoses in the years $2008-2016$. An increasing number of diagnoses were among women and those aged over 35 years. This study suggests that while the number of people being diagnosed with autism is increasing, many are still unrecognised by healthcare services.

\section{Keywords}

autism spectrum disorders, health services, prevalence, screening

\section{Introduction}

Estimates of the prevalence of autism spectrum disorders (hereafter autism) suggest rates of around 1\% in the general population, but with much debate and the suggestion that not all individuals are identified (Brugha et al., 2011, 2016; Chiarotti \& Venerosi, 2020; Fombonne et al., 2021; Lyall et al., 2017). The majority of studies of autism prevalence have been conducted in birth or child cohorts using a variety

\footnotetext{
'Cardiff University, UK

${ }^{2}$ Swansea University, UK

${ }^{3}$ Cardiff and Vale University Health Board, UK

Corresponding author:

Jack FG Underwood, Neuroscience and Mental Health Research

Institute, Division of Psychological Medicine and Clinical

Neurosciences, Cardiff University, Hadyn Ellis Building, Maindy Road,

Cardiff CFIO 3AT, UK.

Email: underwoodj4@cardiff.ac.uk
} 
of epidemiological methodologies (Elsabbagh et al., 2012; Fombonne et al., 2021). These have provided varying estimates with some suggesting increasing prevalence and incidence of autism (Idring et al., 2015; Maenner et al., 2020). This potentially reflects greater societal recognition and awareness of autism beyond historic descriptions associated with intellectual disability to the full spectrum of experience (Elsabbagh et al., 2012; Russell et al., 2015). A recent systematic review and meta-analysis confirmed that in the highest quality studies, a reliable estimate of male to female ratio was around 3:1, although there is increased recognition that women and girls with autism are underserved and unrecognised based on current diagnostic criteria and service provision (Lockwood Estrin et al., 2020; Loomes et al., 2017). Epidemiological studies of prevalence of autism specifically in adults are scarce, limited to pioneering work by Brugha using the UK Adult Psychiatric Morbidity Survey population case-register, a 2019 study using administrative data of autism prevalence in the United States Medicaid Program, and a 2021 study using the UK General Practice Research Database (Brugha et al., 2016; Jariwala-Parikh et al., 2019; Russell et al., 2021).

In Wales, 2016 saw the launch of a new national Integrated Autism Service (IAS) as part of the Autistic Spectrum Disorder Strategic Action Plan from Welsh Government, an example of the development of services to improve provision of autism diagnosis to adults who are more likely to be undiagnosed or experience misdiagnosis (Au-Yeung et al., 2019; Holtom et al., 2019; Lai \& BaronCohen, 2015). The global burden of autism is unknown, but in the United States and the United Kingdom, the annual cost is estimated in billions, and earlier identification and assistance enables adaptation and support while improving social communication and reducing anxiety (Buescher et al., 2014; Jariwala-Parikh et al., 2019; Lai et al., 2014). No prior whole population analyses of autism rates have been performed in Wales, with only estimates based on the Pupil Level School Age Census of Special Educational Needs and a 1988-2004 clinical registry study of children from the Rhondda and Taff Ely areas of South Wales evident in the literature (Holtom et al., 2019; Latif \& Williams, 2007). Establishing incidence data on a population level offers the opportunity to inform future healthcare policy and provision, leading to improvements in quality of life for autistic people across their lifespan. To address this need, we used anonymised healthcare record data from the Secure Anonymised Information Linkage (SAIL) Databank to perform an e-cohort study establishing the incidence and prevalence rates of autism diagnosed in Wales from 2001 to 2016.

\section{Methodology}

\section{Data sources, population and settings}

This was a retrospective population-based electronic cohort study. Data were sourced from the SAIL Databank (www. saildatabank.com) (Lyons et al., 2009). SAIL is a data repository of anonymised person based linkable data from healthcare and public settings. Healthcare in Wales is provided through defined geographical catchment areas, known as Local Health Boards, under the National Health Service (NHS), while social care is planned and commissioned by 22 local government authorities defined by geographic area. All Health Boards utilise an integrated national electronic record system, the Welsh Clinical Portal, while individual general practices (GPs) (primary care) operate their own electronic record systems. A central 'spine' provides personal identifier information to clinical and social care services to integrate identification. Autism diagnostic services prior to 2016 were independently commissioned by Health Boards, usually through paediatric or child and adolescent mental healthcare services for those under 18 years of age, and through community mental health teams for those 18 or older. Clinical encounters in secondary care are routinely recorded within the Welsh Clinical Portal, with letters then sent to individuals' general practitioners and integrated into individuals' notes held on file. Participating services submit their primary care (GP) clinic electronic health records, electronic hospital administration and patient health records, and central health and social care electronic administration records to SAIL, where it is anonymised at inclusion (SAIL Databank - The Secure Anonymised Information Linkage Databank, n.d.). The policies, permissions, processes, structures and controls applied to manage the SAIL databank are described elsewhere (Ford et al., 2009; Jones et al., 2019; Lyons et al., 2009). Data access was approved by the SAIL independent Information Governance Review Panel in 2018 (application 0843 ). No community members were involved in this study.

This study used the Welsh Longitudinal General Practice (WLGP) data set and PEDW (Patient Episode Database for Wales) for extracting relevant diagnostic data, and the Welsh Demographic Service Dataset (WDSD), which includes anonymised demographics, geographic index (LSOA = Lower Layer Super Output Area index 2011) and deprivation data (Welsh Index of Multiple Deprivation quintile 2011). The WDSD is an administrative register of all individuals in Wales to use NHS services, including anonymised demographics and GP registration. The PEDW data set comprises hospital admissions (inpatient and day case) clinical information for all NHS Wales hospitals. At the time of the analysis, 333 (of 432, 77\%) GPs in Wales were supplying their data to SAIL for the WLGP data set, covering $79 \%$ of the population. Anonymised information held in the SAIL Databank includes information pertaining to individuals who have moved into or away from the recruitment areas, or who have been born or died over the study period, reflecting population turnover. In total, records for 3.63 million individuals from the Welsh population over the period 2000-2016 were examined for inclusion in the study. 
Individuals with autism were identified in the WLGP and PEDW data sets using ICD (International Classification of Diseases) version 10 codes and Read Codes v2. This methodology has been validated in previous studies of other psychiatric disorders (Lloyd et al., 2015). The cohort utilised the ICD-10 codes under F84. These codes have been used in previous comparable health record linkage study designs of autism selecting for the maximum sensitivity of autistic phenotypes (Brooks et al., 2021; Idring et al., 2015; Jariwala-Parikh et al., 2019; Skonieczna-Żydecka et al., 2017). The following Read Codes v2 were selected to mirror the ICD-10 diagnostic coding: 1J9., E140., E1400, E1401, E140z, Eu840, Eu841, Eu845, E141., Eu84., Eu842, Eu843, Eu844, Eu845, Eu84y and Eu84z (Appendix 1). Data in the SAIL databank are held in a central repository, requiring extraction and interrogation using structured query language (SQL DB2). SQL code was written to identify eligible individuals from the databank based on presence of the inclusion criteria codes in the WLGP and PEDW data sets. WDSD data were used to prevent multiple counting of cases through location change and for recording of deaths. No deprivation, race/ethnicity or geographic data were extracted for this study. Specific data on socioeconomic status and educational attainment were not available. There were no age or intellectual functioning exclusion criteria for this study.

\section{Statistical analysis}

Analysis of extracted outputs was processed utilising IBM SPSS Statistics 23 for Windows (IBM Corp. Released, 2015) and R version 4.0.3 (R Core Team, 2020) utilising the 'Tidyverse' packages (Wickham et al., 2019). For analysis of recorded incidence and prevalence, the denominator was calculated annually utilising all individuals alive and living in Wales and registered with a GP providing data to SAIL between 1st of January 2000 and 31st of December 2016. Prevalence of recorded autism and annual rates of first ever incident recorded autism were calculated for the whole 16-year period or until death, whichever was earlier. Prevalence was defined as any entry of autism in SAIL records ever, including diagnosis prior to 2000. A new incident episode was defined as an entry in the records with no previous entry of that problem ever recorded and with at least 6 months' worth of WLGP data prior to that diagnosis. Annual first ever incident rates were calculated per person-years at risk, where the proportion of data held for each year for each individual was used to give an accurate estimate of record coverage. Person-time at risk was calculated using the start of each year (1st January) or start of registration (plus 6 months), whichever later. End date was earliest of: date of leaving practice supplying SAIL, date of death or end of year (31st December).

\section{Results}

\section{Autism incidence and prevalence}

The prevalence of autism recorded in electronic health records increased across the study period of 2001-2016, from $0.189(95 \%$ confidence interval $(\mathrm{CI})=0.170-0.210)$ per 1000 people in 2001 to $5.068(95 \% \mathrm{CI}=4.979-5.159)$ per 1000 people in 2016 (Table 1), reflecting cumulative incidence across the data set. Prevalence of autism diagnosis in male electronic healthcare records prevalence rose from $0.324(95 \% \mathrm{CI}=0.289-0.364)$ per 1000 people in 2001 to 7.995 (95\% CI $=7.836-8.156)$ per 1000 people in 2016. Prevalence of autism diagnosis in female healthcare records rose from $0.056(95 \% \mathrm{CI}=0.043-0.074)$ per 1000 people in 2001 to $2.149(95 \% \mathrm{CI}=2.069-2.234)$ per 1000 people in 2016.

The yearly incidence for new recording of autism diagnosis in electronic health records grew from 0.188 (95\% $\mathrm{CI}=0.169-0.209)$ per 1000 person-years in 2001, up to a maximum of $0.644(95 \% \mathrm{CI}=0.612-0.677)$ per 1000 person-years in 2016 (Table 2), a 3.4-fold increase. This change comprised an increase in recorded incidence in both male and female individuals, from $0.322(95 \%$ $\mathrm{CI}=0.286-0.361)$ and $0.057(95 \% \mathrm{CI}=0.043-0.075)$ per 1000 person-years at risk up to $0.945(95 \% \mathrm{CI}=0.891-$ $1.003)$ and $0.345(95 \% \mathrm{CI}=0.313-0.380)$ per 1000 person-years, respectively, for 2016.

The number of new incident recorded diagnosis of autism on males' healthcare records in SAIL increased by 3.94 times over the study period, from 282 cases on 2001 records to 1110 cases on 2016 records (Table 2). This increase was half the eightfold rate increase seen among female healthcare records was double the increase seen in males, from 51 new diagnoses recorded in 2001 to 408 in 2016. This coincided with an increase in the number of diagnoses made in adulthood. In 2001, 19 (6.74\%) males over the age of 35 years had a new diagnosis of autism recorded on their healthcare record, by 2016 this had increased to $100(9.01 \%)$ males over the age of 35 years with a new diagnosis of autism recorded in that year (Figure 1). This trend was seen across both sexes.

\section{Discussion}

In this large population-based electronic cohort study, we draw on anonymised healthcare record data from GP and hospital encounters for over 3.6 million individuals to produce the first national all-age estimates of recorded autism prevalence and incidence for Wales. We found a recorded prevalence of autism of $5.068(95 \% \mathrm{CI}=4.979-5.159)$ per 1000 people in 2016 equivalent to $0.51 \%$ of the population, and a 3.4-fold increase in recorded autism incidence over the study period from $0.188(95 \% \mathrm{CI}=0.169-0.209)$ per 1000 person-years in 2001, up to a maximum of 0.644 $(95 \% \mathrm{CI}=0.612-0.677)$ per 1000 person-years in 2016 . 


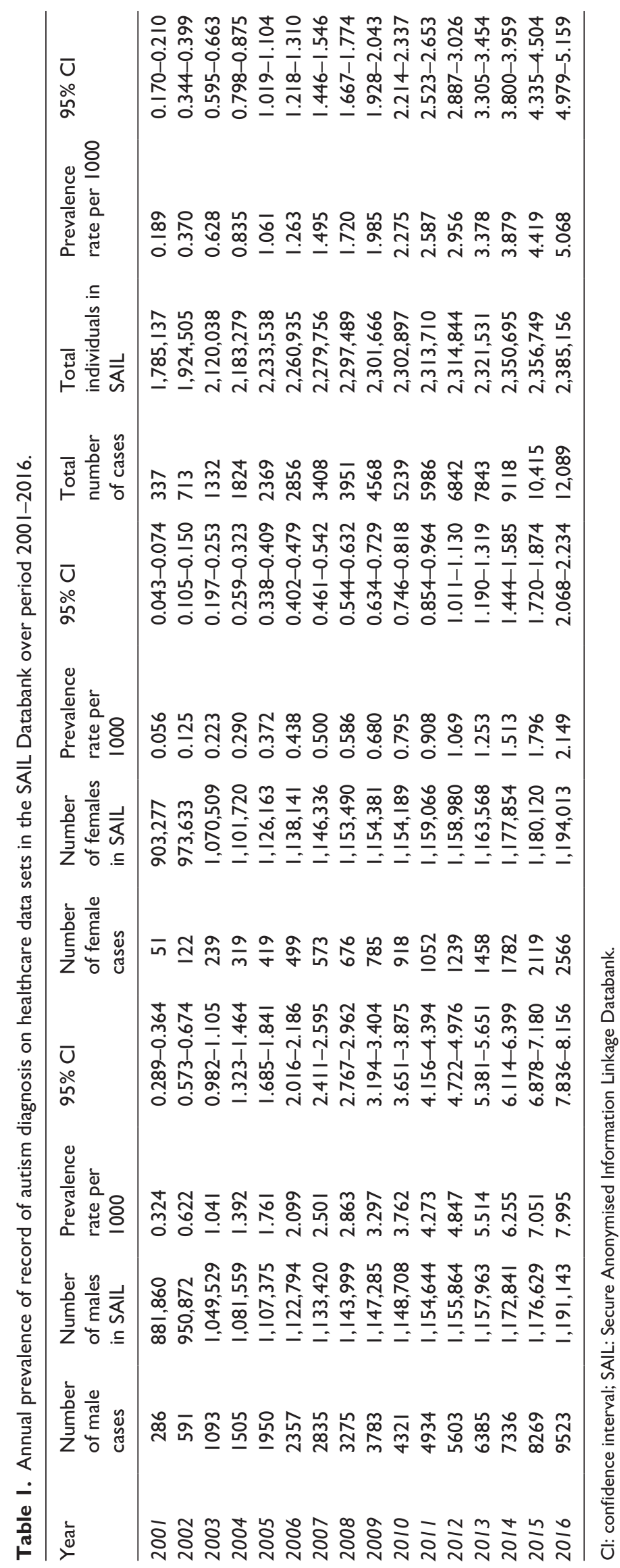




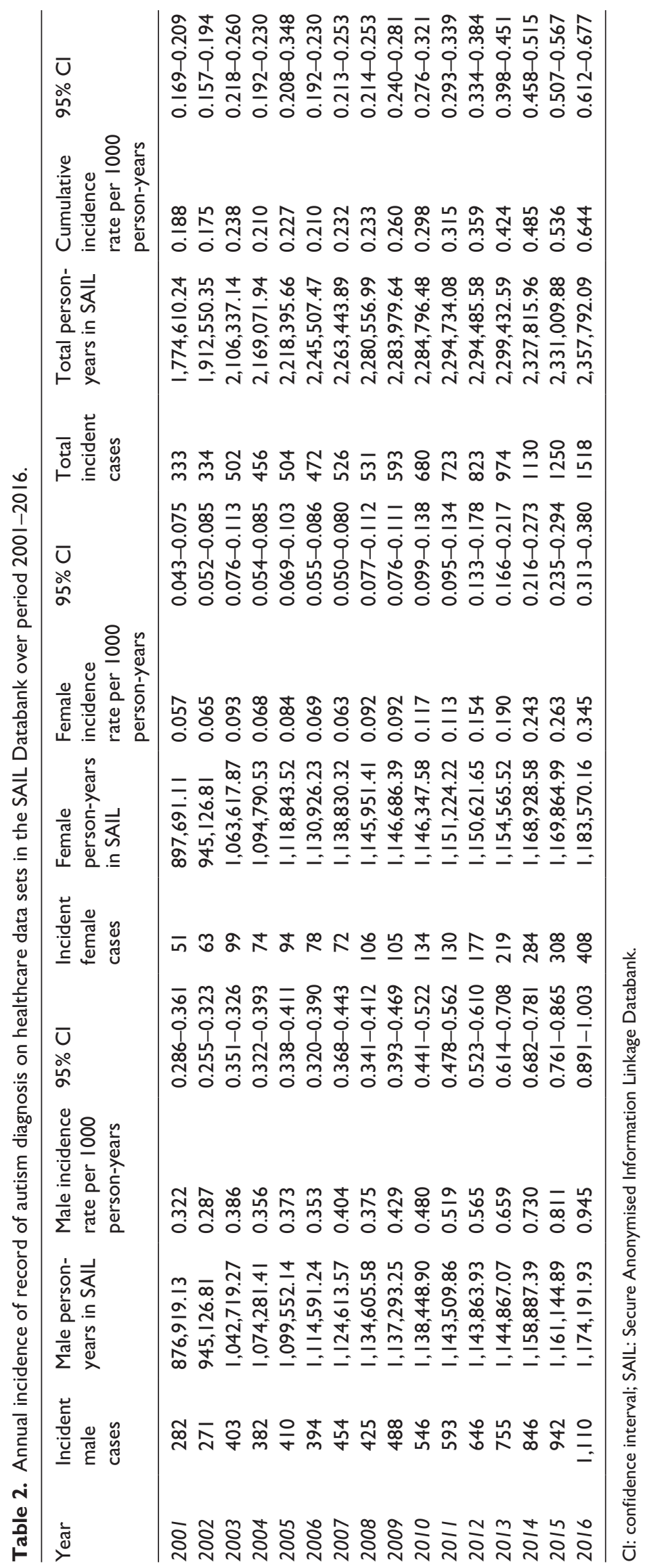




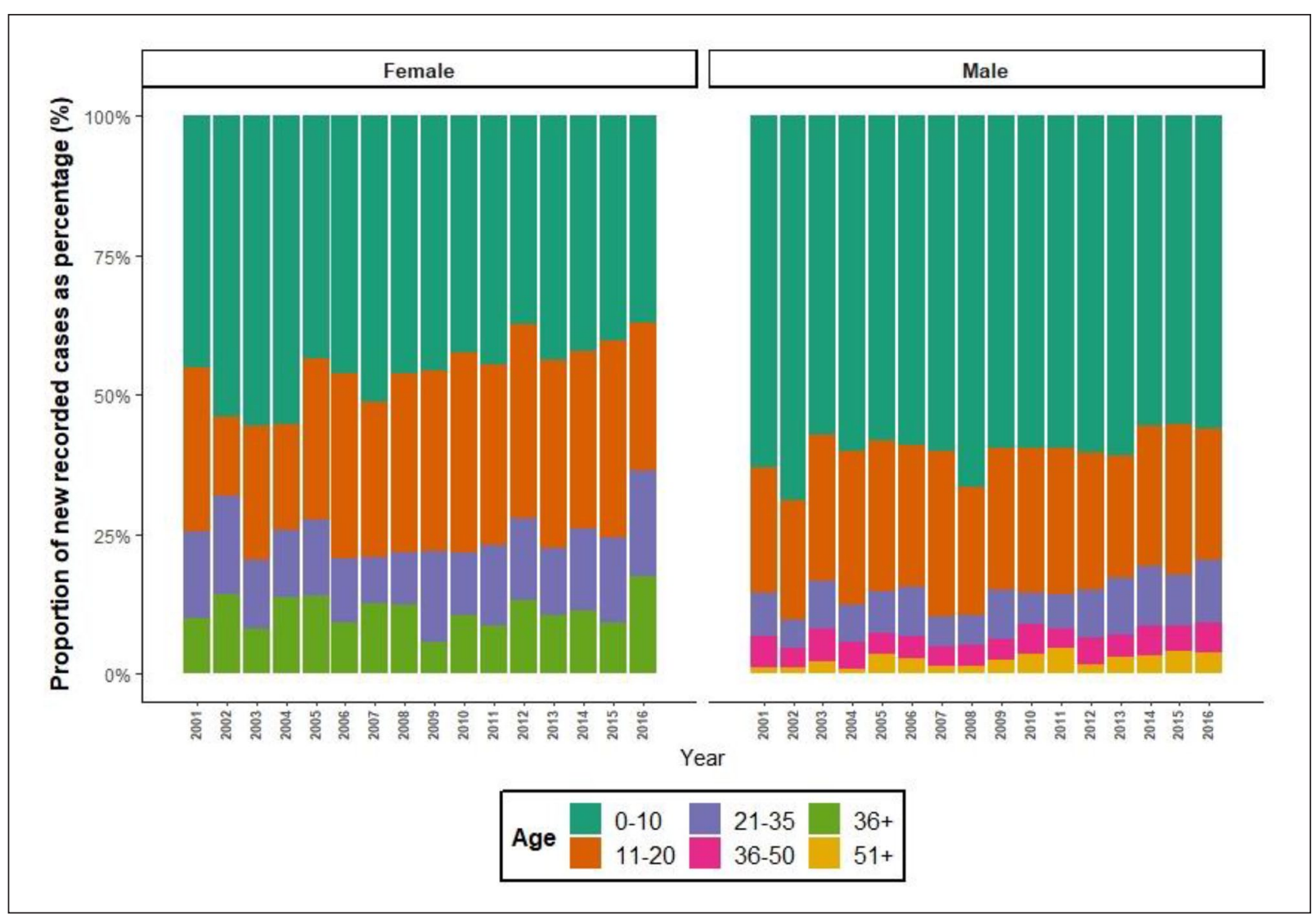

Figure I. Proportion of incident recorded cases divided by sex and age grouping over assessed years (200I-20I6).

Number of new-recorded cases of autism recorded on healthcare record plotted by sex and age grouping plotted over assessed period (200I-2016) in SAIL Databank. Age groupings to meet minimum sample size reporting guidelines for SAIL Databank.

We demonstrate an increase in recorded incidence of autism diagnosis, particularly in the 8-year period from 2008 to 2016. During this time, new-recorded diagnoses increased by $>150 \%$. Despite this, the absolute recorded incidence rate remains low. Multiple groups have reported that rates of autism diagnosis are increasing over time (Lai et al., 2014; Russell et al., 2015). A previous study of comparative data from the General Practice Research Database (GPRD) demonstrated that the incidence rate among children aged 8 years during an overlapping time period (20012010) remained static; however, a more recent analysis across the whole lifespan and for a wider time period (1998-2018) in the same data set by Russell et al. found that there was a $787 \%$ exponential increase in recorded incidence of autism diagnoses (Russell et al., 2021; Taylor et al., 2013). The authors of the 2021 study found the increase in diagnoses was greater in females than in males and concluded that this was likely due to greater awareness and availability of diagnosis (Russell et al., 2021). We would therefore suspect that the increase in incident recorded diagnoses in this study represents better identification and provision for diagnosis of autism in the healthcare system. This particularly noticeable among the older population and women, where there has been considerable systematic diagnostic bias, again replicating Russell et al.'s findings (Loomes et al., 2017; Russell et al., 2021). We observed a trend towards more diagnoses being made among adults over 35 years, and there were eight times more women diagnosed with autism in 2016 than in 2001. We, like others, would suggest that this increase is due to recognition, increased societal awareness, changes in practice and improved availability of diagnostic services particularly for adults with autism (Brugha et al., 2016; Elsabbagh et al., 2012; Russell et al., 2015).

Estimates point to a prevalence of autism in the general population around 1\% (Abdallah et al., 2011; Baio et al., 2018; Chiarotti \& Venerosi, 2020; Fombonne et al., 2021; Lord et al., 2020; Lyall et al., 2017; Russell et al., 2015; Taylor et al., 2013). Prior studies of autism prevalence have examined a number of birth or school cohorts, CDC surveillance data on children's health records in the United States and the GPRD for GP health records of children in the United Kingdom (Baio et al., 2018; Elsabbagh et al., 2012; Fuentes et al., 2020; Russell et al., 2015; Taylor et al., 2013; Thomaidis et al., 2020). Brugha et al. were reportedly the first to ascertain data for adults, finding a prevalence of $0.98 \%-1.1 \%$ using a national stratified survey methodology (Brugha et al., 2011, 2016). Further studies have corroborated the 
$\sim 1 \%$ estimate, with Fombonne et al. (2021) review paper citing a median prevalence in 26 high-income countries of 0.97\% (Chiarotti \& Venerosi, 2020; Fombonne et al., 2021). Our prevalence estimate of 5.068 (95\% CI $=4.979-5.159)$ per 1000 people is lower than these epidemiological studies. This may be due to methodological differences reflecting the under-identification and under-reporting of autism in healthcare records, or due to differences of sample population; most published autism prevalence studies are in children, with few studies of autism prevalence in adults or across the lifespan as in this study.

Our prevalence figure is more comparable to other healthcare record and administrative data analyses. Of these Jariwala-Parikh et al.'s 2019 study of autism prevalence in the US Medicaid programme is one of the only few examining adults, finding an annual prevalence of autism diagnosis of 3.66 per 1000 in 2008 (Jariwala-Parikh et al., 2019). Studies in Europe using administrative data and with healthcare systems similar to Wales have produced prevalence estimates of between 3.5 and 5.8/1000 children in Poland, 3.8/1000 in Germany, 3.6/1000 in France, 5.8 and 15/1000 in Greece, 4.0 and 24.6/1000 in Sweden and 5.9/1000 in Spain (Bachmann et al., 2016; Delobel-Ayoub et al., 2015; Fuentes et al., 2020; Idring et al., 2015; Skonieczna-Żydecka et al., 2017; Thomaidis et al., 2020). The majority of these studies were conducted in child cohorts, and therefore, it would be expected prevalence would increase with annual incidence of new diagnosis into adulthood. In our cohort, variance due to population turnover means that while annual recorded autism prevalence increases, this is not equal to the growth of annual incidence. Annual recorded incidence likely exceeds any change in autism population prevalence, therefore demonstrating under-identification and reporting.

This study utilised the SAIL Databank, a nationally representative, high-quality anonymised e-cohort of $>3.6$ million individuals' records (Lyons et al., 2009). We reviewed General Practice and Hospital Encounter data sets, comprising ICD-10 and Read v2 Codes for diagnoses under analysis. As an e-cohort study, exposure and outcome classification errors cannot be excluded as we are unable to verify the diagnoses. Electronic records of GP data prior to 2000 are sparsely held in the SAIL Databank, and therefore, prevalent cases prior to 2000 are likely missing from this estimate. This explains the dramatic increase from very low baseline of our reported prevalence. It is likely that this study misses individuals with autism in the community who have not come into contact with healthcare services resulting in their diagnosis not being on record. Prior to 2016 autism diagnostic services in Wales were organised by individual Health Boards, and disparate in availability and structure (Holtom et al., 2019). Anecdotal reports suggest many adults sought private diagnoses due to lack of provision, which may not have reported findings into NHS healthcare services. This study is therefore likely to be an underestimation. Diagnostic overshadowing, the practice of one diagnosis taking primacy and leading to underdiagnosis of co-occurring conditions, is also recognised to be a problem within autism (Hollocks et al., 2018). This study used the ICD-10 code F84 to define cases of autism, along with comparable identifiers from Read Codes v2. This methodology has been used by comparable studies to maximise sensitivity to the autism phenotype; however, it does include diagnoses such as Rett's syndrome which are syndromes associated with specific causal factors which may not be representative of autism as a whole (Idring et al., 2015; Jariwala-Parikh et al., 2019; Skonieczna-Żydecka et al., 2017). The definition of autism case status remains a challenge among epidemiological studies, with no uniform method among published studies (Fombonne et al., 2021). These limitations may compound to generate errors in our estimates and contribute to lower figures, although our large sample size and replication of comparable results would suggest these errors are minimal. We have attempted to compensate through use of both primary and secondary care data, and these limitations are common to e-cohort and record linkage studies which depend upon individuals' attendance, recognition and recording to be accurate.

In conclusion, in our national population sample, we found an increase in annual recorded incidence over the study period to 2016. This included increases in diagnosis of females and those over age 35 years. This is consistent with improved societal recognition and access to diagnostic services resulting in greater rates of diagnosis; incident cases not picked up in childhood being diagnosed as adults. Despite this, we found lower prevalence rates than demonstrated in previous epidemiological survey and birth cohort studies, comparable to other health record and administrative study methodologies, suggesting that a large proportion of those adults with autism are not recognised by the healthcare system. Increased rates of diagnosis and more prevalent autism in the community necessitate increased funding for specialist services to enable autistic adults to receive any support they require.

\section{Author contributions}

J.F.G.U. principally designed the study, acted as principal investigator, statistically analysed the data and wrote the first draft of the manuscript with input from A.F. A.F., M.D.P.-B., A.J. and J.H. all contributed to the design of the study. A.F. and M.D.P.-B. co-assisted with data retrieval and analysis. All authors critically read the manuscript and contributed to the data interpretation and writing. All authors approved the final version of the manuscript. The study protocol and summary data may be requested from J.F.G.U.

\section{Declaration of conflicting interests}

The author(s) declared the following potential conflicts of interest with respect to the research, authorship and/or publication of 
this article: J.H. has received grants from Takeda for research work unrelated to this project. The other authors declare no competing interests.

\section{Funding}

The author(s) disclosed receipt of the following financial support for the research, authorship and/or publication of this article: This project was funded by a Wellcome Trust ISSF Clinical Primer award, and subsequent Wellcome Trust GW4-CAT Clinical Doctoral Fellowship (222849/Z/21/Z) to J.F.G.U. J.H., A.J., M.D.P.-B. and J.F.G.U. are supported by Healthcare Research Wales through the National Centre for Mental Health (NCMH) (CA04). J.H. and J.F.G.U. are supported by an MRC Pathfinder Grant (MC_PC_17212), and M.D.P.-B., A.F. and A.J. are supported by an MRC Pathfinder Grant (MC_PC_17211).

\section{ORCID iD}

Jack FG Underwood (iD https://orcid.org/0000-0003-1731-6039

\section{References}

Abdallah, M. W., Greaves-Lord, K., Grove, J., NørgaardPedersen, B., Hougaard, D. M., \& Mortensen, E. L. (2011). Psychiatric comorbidities in autism spectrum disorders: Findings from a Danish Historic Birth Cohort. European Child and Adolescent Psychiatry, 20(11-12), 599-601. https://doi.org/10.1007/s00787-011-0220-2

Au-Yeung, S. K., Bradley, L., Robertson, A. E., Shaw, R., Baron-Cohen, S., \& Cassidy, S. (2019). Experience of mental health diagnosis and perceived misdiagnosis in autistic, possibly autistic and non-autistic adults. Autism, 23(6), 1508-1518. https://doi.org/10.1177/1362361318818167

Bachmann, C. J., Gerste, B., \& Hoffmann, F. (2018). Diagnoses of autism spectrum disorders in Germany: Time trends in administrative prevalence and diagnostic stability. Autism, 22(3), 283-290. https://doi.org/10.1177/1362361316673977

Baio, J., Wiggins, L., Christensen, D. L., Maenner, M. J., Daniels, J., Warren, Z., Kurzius-Spencer, M., Zahorodny, W., Rosenberg, C. R., White, T., Durkin, M. S., Imm, P., Nikolaou, L., Yeargin-Allsopp, M., Lee, L. C., Harrington, R., Lopez, M., Fitzgerald, R. T., Hewitt, A., . . . Dowling, N. F. (2018). Prevalence of autism spectrum disorder among children aged 8 Years - Autism and developmental disabilities monitoring network, 11 Sites, United States, 2014. MMWR Surveillance Summaries, 67(6), 1-23. https://doi. org/10.15585/mmwr.ss6706a1

Brooks, J. D., Arneja, J., Fu, L., Saxena, F. E., Tu, K., Pinzaru, V. B., Anagnostou, E., Nylen, K., Saunders, N. R., Lu, H., McLaughlin, J., \& Bronskill, S. E. (2021). Assessing the validity of administrative health data for the identification of children and youth with autism spectrum disorder in Ontario. Autism Research, 14(5), 1037-1045. https://onlinelibrarywiley-com.abc.cardiff.ac.uk/doi/full/10.1002/aur.2491

Brugha, T. S., McManus, S., Bankart, J., Scott, F., Purdon, S., Smith, J., Bebbington, P., Jenkins, R., \& Meltzer, G. C. W. (2011). Epidemiology of autism spectrum disorders in adults in the community in England. Archives of General Psychiatry, 68(5), 459-465. https://doi.org/10.1001/archgenpsychiatry.2011.38
Brugha, T. S., Spiers, N., Bankart, J., Cooper, S. A., McManus, S., Scott, F. J., Smith, J., \& Tyrer, F. (2016). Epidemiology of autism in adults across age groups and ability levels. British Journal of Psychiatry, 209(6), 498-503. https://doi. org/10.1192/bjp.bp.115.174649

Buescher, A. V. S., Cidav, Z., Knapp, M., \& Mandell, D. S. (2014). Costs of autism spectrum disorders in the United Kingdom and the United States. JAMA Pediatrics, 168(8), 721-728. https://doi.org/10.1001/JAMAPEDIATRICS.2014.210

Chiarotti, F., \& Venerosi, A. (2020). Epidemiology of autism spectrum disorders: A review of worldwide prevalence estimates since 2014. Brain Sciences, 10(5), 274. https://doi. org/10.3390/brainsci10050274

Delobel-Ayoub, M., Ehlinger, V., Klapouszczak, D., Maffre, T., Raynaud, J.-P., Delpierre, C., \& Arnaud, C. (2015). Socioeconomic disparities and prevalence of autism spectrum disorders and intellectual disability. PLOS ONE, 10 (11), e0141964. https://doi.org/10.1371/JOURNAL.PONE. 0141964

Elsabbagh, M., Divan, G., Koh, Y. J., Kim, Y. S., Kauchali, S., Marcín, C., Montiel-Nava, C., Patel, V., Paula, C. S., Wang, C., Yasamy, M. T., \& Fombonne, E. (2012). Global prevalence of autism and other pervasive developmental disorders. Autism Research, 5(3), 160-179. http://www. autismepidemiology.net

Fombonne, E., MacFarlane, H., \& Salem, A. C. (2021). Epidemiological surveys of ASD: Advances and remaining challenges. Journal of Autism and Developmental Disorders, 51, 4271-4290. https://doi.org/10.1007/s10803021-05005-9

Ford, D. V., Jones, K. H., Verplancke, J.-P., Lyons, R. A., John, G., Brown, G., Brooks, C. J., Thompson, S., Bodger, O., Couch, T., \& Leake, K. (2009). The SAIL databank: Building a national architecture for e-health research and evaluation. BMC Health Services Research, 9(1), Article 157. https://doi.org/10.1186/1472-6963-9-157

Fuentes, J., Basurko, A., Isasa, I., Galende, I., Muguerza, M. D., García-Primo, P., García, J., Fernández-Álvarez, C. J., Canal-Bedia, R., Posada de la Paz, M., \& de la Paz, M. P. (2020). The ASDEU autism prevalence study in northern Spain. European Child \& Adolescent Psychiatry, 30(4), 579-589. https://link-springer-com.abc.cardiff.ac.uk/article/10.1007/s00787-020-01539-y

Hollocks, M. J., Lerh, J. W., Magiati, I., Meiser-Stedman, R., \& Brugha, T. S. (2018). Anxiety and depression in adults with autism spectrum disorder: A systematic review and metaanalysis. Psychological Medicine, 49, 559-572. https://doi. org/10.1017/S0033291718002283

Holtom, D., Lloyd-Jones, S., \& Prosser, N. (2019). Scoping study for the alignment and development of autism and neurodevelopmental services. https:/gov.wales/autism-and-neurodevelopmental-services

IBM Corp. Released. (2015). IBM SPSS Statistics for Windows, Version 23.0.

Idring, S., Lundberg, M., Sturm, H., Dalman, C., Gumpert, C., Rai, D., Lee, B. K., \& Magnusson, C. (2015). Changes in prevalence of autism spectrum disorders in 2001-2011: Findings from the Stockholm Youth Cohort. Journal of Autism and Developmental Disorders, 45(6), 1766-1773. https://doi.org/10.1007/s10803-014-2336-y 
Jariwala-Parikh, K., Barnard, M., Holmes, E. R., West-Strum, D., Bentley, J. P., Banahan, B., \& Khanna, R. (2019). Autism prevalence in the medicaid program and healthcare utilization and costs among adult enrollees diagnosed with autism. Administration and Policy in Mental Health and Mental Health Services Research, 46(6), 768-776. https:// doi.org/10.1007/s10488-019-00960-z

Jones, K. H., Ford, D. V., Thompson, S., \& Lyons, R. (2019). A profile of the SAIL databank on the UK secure research platform. International Journal of Population Data Science, 4(2). https://doi.org/10.23889/IJPDS.V4I2.1134

Lai, M. C., \& Baron-Cohen, S. (2015). Identifying the lost generation of adults with autism spectrum conditions. The Lancet Psychiatry, 2(11), 1013-1027. https://doi.org/10.1016/ S2215-0366(15)00277-1

Lai, M. C., Lombardo, M. V., \& Baron-Cohen, S. (2014). Autism. The Lancet, 383(9920), 896-910. https://doi.org/10.1016/ S0140-6736(13)61539-1

Latif, A. H. A., \& Williams, W. R. (2007). Diagnostic trends in autistic spectrum disorders in the South Wales valleys. Autism, 11(6), 479-487. https://doi.org/10.1177/1362361307083256

Lloyd, K., McGregor, J., John, A., Craddock, N., Walters, J. T., Linden, D., Jones, I., Bentall, R., Lyons, R. A., Ford, D. V., \& Owen, M. J. (2015). A national population-based e-cohort of people with psychosis (PsyCymru) linking prospectively ascertained phenotypically rich and genetic data to routinely collected records: Overview, recruitment and linkage. Schizophrenia Research, 166(1-3), 131-136. https://doi.org/10.1016/j.schres.2015.05.036

Lockwood Estrin, G., Milner, V., Spain, D., Happé, F., \& Colvert, E. (2020). Barriers to autism spectrum disorder diagnosis for young women and girls: A systematic review. Review Journal of Autism and Developmental Disorders, 8 , 454-470. https://doi.org/10.1007/s40489-020-00225-8

Loomes, R., Hull, L., \& Mandy, W. P. L. (2017). What is the male-to-female ratio in autism spectrum disorder? A systematic review and meta-analysis. Journal of the American Academy of Child and Adolescent Psychiatry, 56(6), 466474. https://doi.org/10.1016/j.jaac.2017.03.013

Lord, C., Traolach, S. B., Tony, C., James, C., Guillaume, D., Thomas, F., Emily, J. H. J., Rebecca, M. J., Andrew, P., Matthew, W. S., Julie, L. T., \& Jeremy, V.-V. (2020). Autism spectrum disorder. Nature Reviews, 6(5), 1-23. https://doi.org/10.1038/s41572-019-0138-4

Lyall, K., Croen, L., Daniels, J., Fallin, M. D., Ladd-Acosta, C., Lee, B. K., Park, B. Y., Snyder, N. W., Schendel, D., Volk, H., Windham, G. C., \& Newschaffer, C. (2017). The changing epidemiology of autism spectrum disorders. Annual Review of Public Health, 38, 81-102. https:// www. annualreviews.org/doi/abs/10.1146/annurev-publhealth-031816-044318

Lyons, R. A., Jones, K. H., John, G., Brooks, C. J., Verplancke, J.-P. P., Ford, D. V., Brown, G., \& Leake, K. (2009). The SAIL databank: Linking multiple health and social care datasets. BMC Medical Informatics and Decision Making, 9(1), Article 3. https://doi.org/10.1186/14726947-9-3

Maenner, M. J., Shaw, K. A., Baio, J., Washington, A., Patrick, M., DiRienzo, M., Christensen, D. L., Wiggins, L. D., Pettygrove, S., Andrews, J. G., Lopez, M., Hudson, A.,
Baroud, T., Schwenk, Y., White, T., Rosenberg, C. R., Lee, L. C., Harrington, R. A., Huston, M., . . . Dietz, P. M. (2020). Prevalence of autism spectrum disorder among children aged 8 Years-Autism and developmental disabilities monitoring network, 11 Sites, United States, 2016. MMWR Surveillance Summaries, 69(4), 1-12. https://doi. org/10.15585/MMWR.SS6904A1

R Core Team. (2020). $R$ : A language and environment for statistical computing. https://www.r-project.org/

Russell, G., Collishaw, S., Golding, J., Kelly, S. E., \& Ford, T. (2015). Changes in diagnosis rates and behavioural traits of autism spectrum disorder over time. BJPsych Open, 1(2), 110-115. https://doi.org/10.1192/bjpo.bp.115.000976

Russell, G., Stapley, S., Newlove-Delgado, T., Salmon, A., White, R., Warren, F., Pearson, A., \& Ford, T. (2021). Time trends in autism diagnosis over 20 years: A UK population-based cohort study. Journal of Child Psychology and Psychiatry. Advance online publication. https://doi. org/10.1111/JCPP.13505

SAIL Databank - The Secure Anonymised Information Linkage Databank. (n.d.). https://saildatabank.com/about-us/datalinkage/

Skonieczna-Żydecka, K., Gorzkowska, I., Pierzak-Sominka, J., \& Adler, G. (2017). The prevalence of autism spectrum disorders in West Pomeranian and Pomeranian Regions of Poland. Journal of Applied Research in Intellectual Disabilities, 30(2), 283-289. https://doi.org/10.1111/JAR.12238

Taylor, B., Jick, H., \& MacLaughlin, D. (2013). Prevalence and incidence rates of autism in the UK: Time trend from 20042010 in children aged 8 years. BMJ Open, 3(10). https://doi. org/10.1136/bmjopen-2013-003219

Thomaidis, L., Mavroeidi, N., Richardson, C., Choleva, A., Damianos, G., Bolias, K., \& Tsolia, M. (2020). Autism spectrum disorders in Greece: Nationwide prevalence in 10-11 year-old children and regional disparities. Journal of Clinical Medicine, 9(7), 2163.

Wickham, H., Averick, M., Bryan, J., Chang, W., McGowan, L. D., François, R., Grolemund, G., Hayes, A., Henry, L., Hester, J., Kuhn, M., Pedersen, T. L., Miller, E., Bache, S. M., Müller, K., Ooms, J., Robinson, D., Seidel, D. P., Spinu, V., \& Yutani, H. (2019). Welcome to the Tidyverse. Journal of Open Source Software, 4(43), 1686. https://doi. org/10.21105/joss.01686

\section{Appendix I}

\section{Coding utilised for identification of autism spectrum disorder phenotype in SAIL}

\section{International Classification of Diseases-Tenth Revision}

- F84 - Pervasive developmental disorders, containing:

- F84.0 - Childhood autism

- F84.1 - Atypical autism

- F84.2 - Rett's syndrome

- F84.3 - Other childhood disintegrative disorder

- F84.4 - Overactive disorder associated with mental retardation and stereotyped movements 
- F84.5 - Asperger's syndrome

- F84.8 - Other pervasive developmental disorders

- F84.9 - Pervasive developmental disorders, unspecified

\section{Read Codes v2.}

The following Read Codes v2 were selected to mirror the ICD-10 diagnostic coding:

- E140. - Autism/infantile autism

- E1400 - Active infantile autism

- E1401 - Residual infantile autism

- E140z - Infantile autism NOS
- Eu840 - Childhood autism/ infantile autism

- Eu841 - Atypical autism

- Eu845 - Asperger's syndrome

- 1J9. - Suspected autism

- E141. - Childhood disintegrative disorder

- Eu84. - Pervasive developmental disorder

- Eu842 - Rett's syndrome

- Eu843 - Other childhood disintegrative disorder

- Eu844 - Overactive disorder associated with mental retardation and stereotyped movements

- Eu845 - Asperger's syndrome

- Eu84y - Other pervasive developmental disorder

- Eu84z-Pervasive developmental disorder, unspecified 